\title{
QCD and Electroweak Boson Production in the Forward Region in $\mathrm{LHCb}^{\S}$
}

\author{
Florin Maciuc* ${ }^{*}$ on Behalf of the LHCb Collaboration
}

\author{
Horia Hulubei National Institute of Physics and Nuclear Engineering, Reactorului 30, P.O. Box MG-6, RO-077125, \\ Bucharest Magurele, EU, Romania
}

\begin{abstract}
Among most recent $\mathrm{LHCb}$ measurements relevant in the context of low-x physics include forward energy flow, $\mathrm{Z}$ and $\mathrm{W}$ cross-section measurements. The energy flow measurements map the forward $\mathrm{LHCb}$ acceptance for four classes of events, namely inclusive minimum bias, non-diffractive, diffractive and hard- $p_{T}$ collisions. Estimates from highenergy collision generators - i.e. various PYTHIA tunes and cosmic-ray generators - were compared to the measurement values. $Z \rightarrow \mu^{+} \mu^{-}$and $W^{ \pm} \rightarrow \mu^{ \pm} v(\bar{v})$ production is measured based on data collected at $7 \mathrm{TeV}$, results are shown differentially and compared to theoretical pQCD predictions.

PACS number(s): 25.75.Dw, 13.85.-t, 12.38.Bx, 12.38.Cy, 12.38.-t.
\end{abstract}

Keywords: Cosmic ray collision generators, detector, electroweak interaction, energy flow, forward physics, hard scattering, minimum bias, Monte Carlo models, parton density functions, pQCD, Pythia generator, QCD.

\section{INTRODUCTION}

The energy flow observed in high energy pp-collision events, probes besides the hard parton-parton collision contribution, also the soft component of the underlying event. At the LHC energies, contributions from Multi-Parton Interaction (MPI) events become important as the soft parton-parton collision has enough energy to produce some of the detector-reconstructed particles [1]. Measurements of the energy flow at large pseudorapidity, $\eta^{1}$, are expected to be sensitive to the parton radiation and MPI contributions [2]. Z, W and low mass Drell-Yan production constitute an important test of Standard Model at LHC energies. Theoretical predictions are available at NNLO with uncertainties of a few percent, and these are dominated by the knowledge of the parton density function (PDF). The $\mathrm{LHCb}$ detector is fully instrumented in the range $\eta \in[2,5]$, hence it can provide input to constrain the PDFs in a range which is not accessible to ATLAS and CMS.

In case of measurements with $\mathrm{Z}$ and $\mathrm{W}$ bosons, these are sensitive to Bjorken-x values down to $1.7 \times 10^{-4}$, and the low mass Drell-Yan measurements at a mass of $5 \mathrm{GeV} / c^{2}$ between $10^{-5}$ and $10^{-3}$. Both ranges cover a kinematic region not investigated by previous experiments.

\footnotetext{
*Address correspondence to this author at the Horia Hulubei National Institute of Physics and Nuclear Engineering, Reactorului 30, P.O.B. MG-6, RO-077125, Bucharest Magurele, EU, Romania; Tel: 0040729152211; Fax: 00400214574440; E-mail: florin.maciuc@cern.ch

${ }^{\S}$ Presented at the Low x workshop, May 30 - June 4 2013, Rehovot and Eilat, Israel.
}

${ }^{1} \eta=-\ln [\tan (\theta / 2)]$ where $\theta$ being the polar angle.

\section{LHCb DETECTOR}

The LHCb detector [3] is a single-arm forward spectrometer covering the pseudorapidity range $2<\eta<5$, designed for the study of particles containing $b$ or $c$ quarks. The detector includes a high-precision tracking system consisting of a silicon-strip vertex locator (VELO) surrounding the $p p$ interaction region, a large-area siliconstrip detector located upstream of a dipole magnet with a bending power of about $4 \mathrm{Tm}$, and three stations of siliconstrip detectors and straw drift tubes placed downstream. The combined tracking system provides a momentum measurement with relative uncertainty that varies from $0.4 \%$ at $5 \mathrm{GeV} / \mathrm{c}$ to $0.6 \%$ at $100 \mathrm{GeV} / \mathrm{c}$, and impact parameter resolution of $20 \mu \mathrm{m}$ for tracks with large transverse momentum. Different types of charged hadrons are distinguished by information from two ring-imaging Cherenkov detectors. Photon, electron and hadron candidates are identified by a calorimeter system consisting of scintillating-pad and preshower detectors, an electromagnetic calorimeter and a hadronic calorimeter. Muons are identified by a system composed of alternating layers of iron and multiwire proportional chambers. The trigger consists of a hardware stage, based on information from the calorimeter and muon systems, followed by a software stage, which applies a full event reconstruction.

The system is perfectly suited to detect and select the final state muons originating in $\mathrm{Z} / \mathrm{W}$ boson decays and low mass Drell-Yan processes in the forward region. To acquire the interesting events, the trigger uses two trigger lines, a single high- $p_{T}$ muon line for $\mathrm{Z} / \mathrm{W}$ production studies and alternatively a dimuon line for the selection of Drell-Yan processes. For the energy flow of charged particles measurements a Minimum Bias sample was used where the 
only trigger requirement is at least one track reconstructed per event.

\section{ENERGY FLOW OF CHARGED PARTICLES}

The energy flow measurement [5] is based on a sample of about 6 million Minimum Bias events at $7 \mathrm{TeV}$ p-p collision energy, which corresponds to an integrated luminosity of about $0.1 n b^{-1}$. The sample was recorded during low luminosity LHC runs, and has low pileup conditions. The energy flow of charged particles is based on tracks with track segments before and after the magnet with very precise momentum determination. Hence, only the long lived charged particles are counted in the energy flow
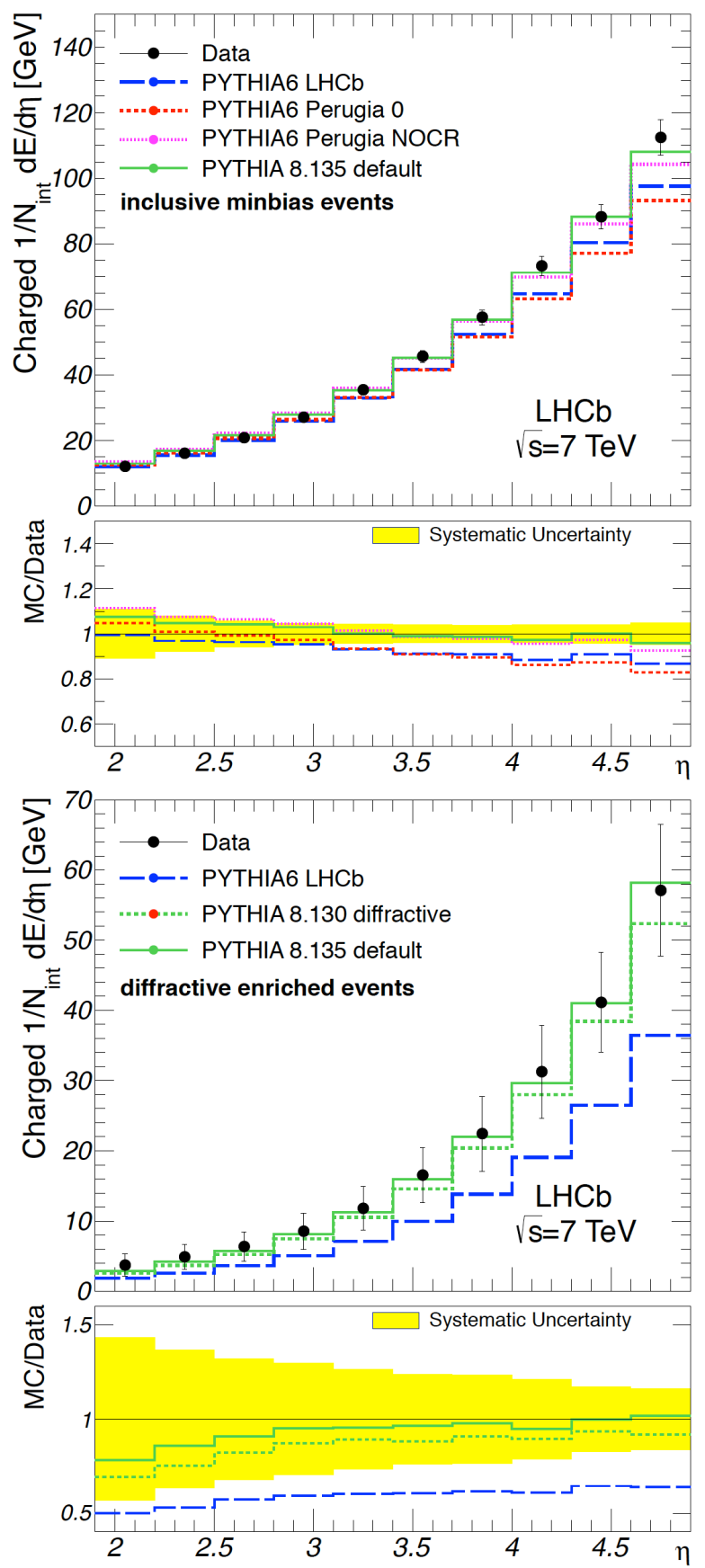

measurements and the measured energy flow normalized over the number of interactions, $N_{i n t}$, is defined: $\frac{1}{N_{\text {int }}} \frac{d E_{\text {charged }}}{d \eta}=\frac{1}{\Delta \eta}\left(\frac{1}{N_{\text {int }}} \sum_{i=1}^{N}{ }_{\text {part }, \eta} E_{i, \eta}\right) \quad$ here $\quad$ the pseudorapidity range is split in 10 equal size bins, $N_{\text {part }, \eta}$ is the total number of particles with $\eta$ in a given bin, and $E_{i, \eta}$ are their energies. Besides the charged component of the energy flow discussed here, the total energy flow has also been estimated by adding the complementary neutral contribution in each bin, for details and values see [5].
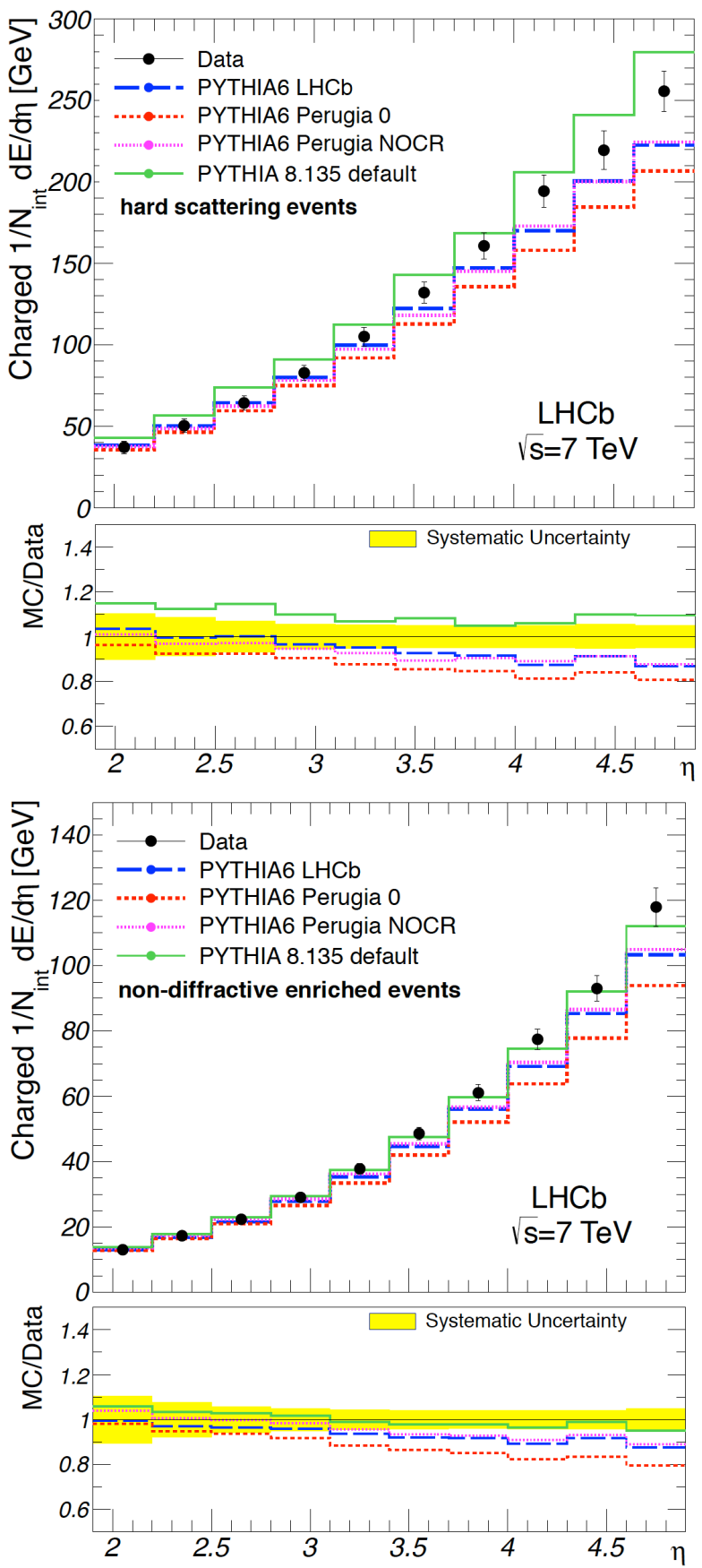

Fig. (1). The efficiency-corrected energy flow measurements of charged particles in four classes of events compared to PYTHIA estimates. 
Events are selected with at least one track with $p \in[2,1000] \mathrm{GeV} / \mathrm{c}$ and with $\eta$ in range $[1.9,4.9]$. Given the VELO backward-forward coverage - as the collision takes place deep inside its volume - it is possible to split events in four classes of inelastic collision events: inclusive minimum bias, non-diffractive, hard scattering and diffractive. The inclusive minimum bias sample is made of all events that passed the previous cuts and it also includes all the events found in the other three samples, too. The hard scattering events are selected by asking for at least one high $p_{T}$ track with $p_{T}>3 \mathrm{GeV} / \mathrm{c}$ in the interval of interest $\eta \in[1.9,4.9]$. The selection of diffractive events is based on a rapidity gap signature [4], by requiring that no track is reconstructed in the backward VELO acceptance of $\eta \in[-3.5,-1.5]$, while for the non-diffractive events at least one track should be reconstructed in this $\eta$ range. Simulation studies give estimated purities for the diffractive and non-diffractive enriched samples to be $70 \%$ and $90 \%$, respectively. Events with more than one reconstructed primary vertex are filtered-out, and the primary vertex position information is not used to avoid biases from primary vertex reconstruction inefficiency.

The measurements are compared to the predictions from various tunes of PYTHIA and four cosmic ray generators. The PYTHIA generator versions include the PYTHIA 6 generator tunes of Perugia 0 and NOCR [6], an LHCb tune [7], and PYTHIA 8.130 plus PYTHIA 8.135 [8] both with the default tune. The cosmic ray generators used are: QGSJET01, QGSJETII-03 [10], EPOS 1.99 [9], and SIBYLL 2.1 [11]. All generators correspond to tunes and versions prior to their tuning to the LHC data. The diffraction processes was not enabled in the Perugia tunes, and in the PYTHIA 8.130 sample only diffractive events were included by filtering the events after generation according to their event type.

To correct for tracking inefficiencies and for particle losses at the edge of the $\mathrm{LHCb}$ acceptance, corrections to the energy flow of charged particles are applied in each bin of $\eta$

These corrections factors are computed by using alternatively: the PYTHIA LHCb-tune, the two Perugia samples and the PYTHIA 8.130 diffractive-only sample. All these samples are fully reconstructed using the $\mathrm{LHCb}$ simulation, digitization, and reconstruction software.

Fig. (1). displays the results for the charged-component of the energy flow, and the overlaid estimates are from PYTHIA-based generators. The uncertainties are systematic only, since the statistical uncertainties are negligible. In computing the systematic errors on the energy flow of charged particles, the following contributions were taken into account: the model dependence of the efficiencies and acceptance correction factors; the tracking efficiency uncertainties; other tracking effects like duplicating the same particle; magnetic field polarity effects; and an uncertainty on the residual pileup component with unreconstructed primary collisions. It can be noted that the relative uncertainty (yellow area in Fig. 1) is the lowest at large pseudorapidity, where measurements are most sensitive to the underlying event.
Generally there is qualitative agreement between data and the PYTHIA estimates, though there are also some clear quantitative differences. Overall the energy flow of charged particles increase with pseudorapidity is steeper in data than for PYTHIA estimates, except for the hard scattering class where the PYTHIA 8.135 results exceed the data measurement values over the entire pseudorapidity range. The latter provides the best description of energy flow of charged particles out of all PYTHIA generators, with the already mentioned exception of the hard scattering class.

Fig. (2), has the same data results as Fig. (1) but the overlaid estimates are provided this time by the cosmic-ray generators. Here the SIBYLL and EPOS models describe much better the data in the soft scattering collisions, where QGSJET models severely overestimate the energy flow of charged particles. This is seen for the inclusive minimum bias and the non-diffractive samples. For hard scattering and diffractive events, all models give about the same degree of agreement or disagreement with respect to $\mathrm{LHCb}$ data. For the diffractive case all four models provide smaller values than data, whereas for hard diffractive events there is no clear preference.

In conclusion none of the generator results can describe all the features seen in data, though SIBYLL, EPOS and to some degree PYTHIA 8.135 come close in reproducing the data.

\section{Z CROSS-SECTION MEASUREMENTS}

For the $Z$ cross-section studies using the $Z \rightarrow \mu^{+} \mu^{-}$ decay channel, the latest $\mathrm{LHCb}$ result [12] is based on data collected at $7 \mathrm{TeV}$ collision energy and correspond to an integrated luminosity of $1 \mathrm{fb}^{-1}$. The cross-section is measured for the following fiducial and kinematic ranges: momenta and pseudorapidities of the two muons $p_{T}>20$ $\mathrm{GeV} / \mathrm{c}$ and $\eta \in[2,4.5]$, and furthermore the invariant dimuon mass must be in $\left[60 \mathrm{GeV} / \mathrm{c}^{2}, 120 \mathrm{GeV} / \mathrm{c}^{2}\right]$. The purity is very high (see Fig. 3a), with contributions to the background from top pairs, $\mathrm{W}$ pairs, and $Z \rightarrow \tau \tau$ decays these estimated from simulation - as well as from misidentified hadrons and heavy flavor $b b$ and $c c$ double semileptonic decays - these contributions estimated directly from data.

The integrated cross-section preliminary result over the previously mentioned fiducial and kinematic range is: $\sigma_{Z \rightarrow \mu \mu}=75.4 \pm 0.3$ (stat) $\pm 1.9($ sys $) \pm 2.6($ lumi $) p b$ which agrees nicely to the fixed order pQCD result at next-to-next-toleading-order (NNLO) of FEWZ [14]:

$$
\sigma_{N N L O}=74.7 \begin{array}{cc}
+1.6 & +0.4 \\
-1.4 & -0.4
\end{array} p
$$

where the first error is due to uncertainty on the PDFs and the second is the theoretical uncertainty. The systematic uncertainties are determined by: uncertainties of tracking, trigger and muon identification efficiencies; magnetic field polarity flip; and the $3.5 \%$ integrated luminosity uncertainty. The MSTW08 PDF - [12] has bibliographic references for used PDF - was used by FEWZ to compute the NNLO 

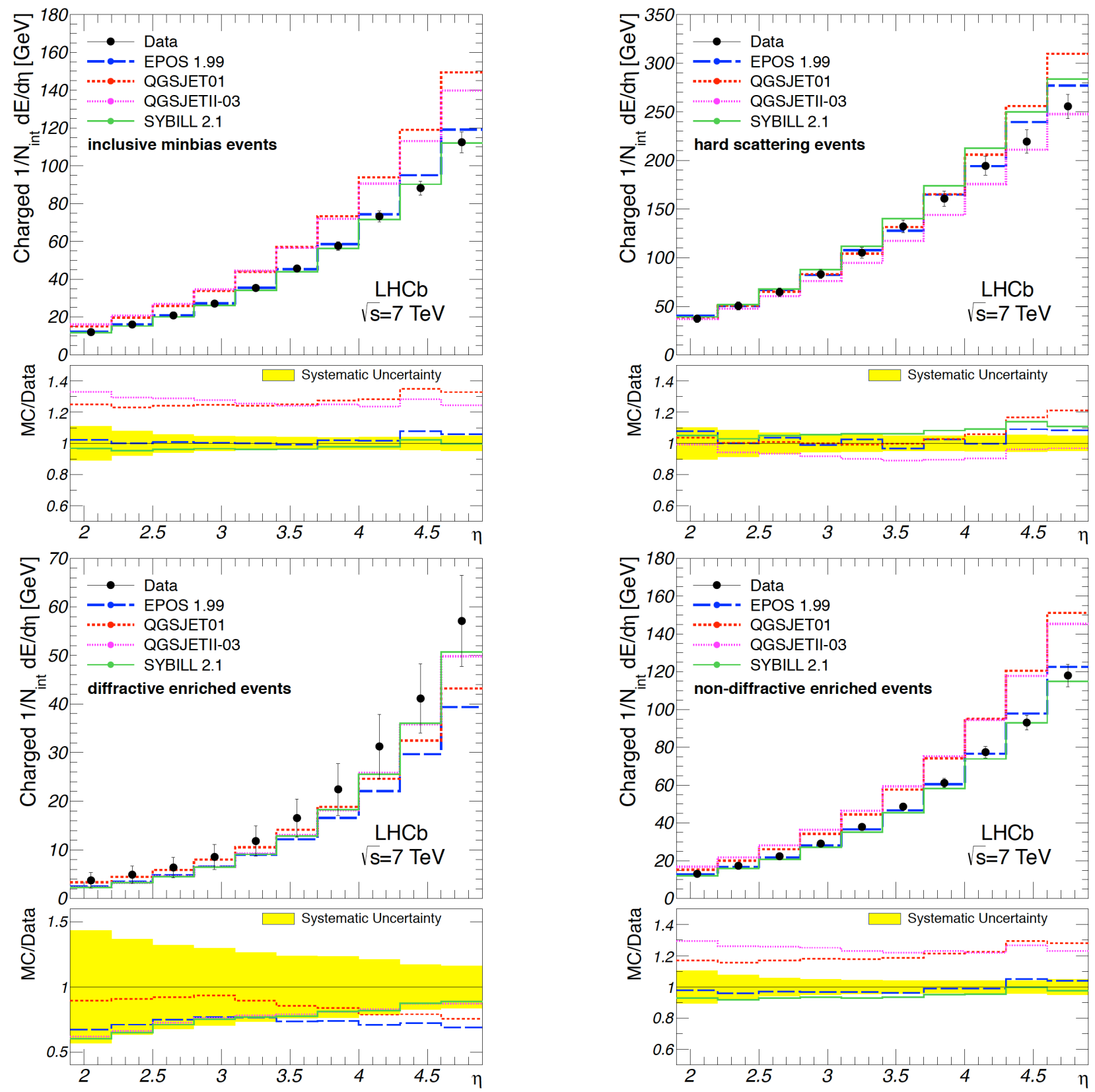

Fig. (2). The efficiency-corrected energy flow measurements of the charged particles in four classes of events and the cosmic ray generator estimates overlaid.

estimate. In Fig. (3b), the $\mathrm{Z}$ production differential crosssection over $Z$ rapidity $\left(y_{Z}\right)$ is compared to NNLO predictions using various PDFs: MSTW08, CT10, NNPDF23, and ABM11 - see [12]. There is an excellent agreement between measurements and theory in this rapidity spectrum of the $\mathrm{Z}$ bosons.

The differential distributions as functions of $p_{T}$ and $\phi^{*}$ variables $^{2}$ of the $Z$ boson are compared to the NNLO FEWZ results and the results from RESBOS and POWHEG. If FEWZ uses a fixed order pQCD approximation at NNLO,

${ }^{2} \varphi^{*}=\tan \left(\varphi_{\text {acop }} / 2\right) / \cosh (\Delta \eta / 2)$ with $\varphi_{\text {acop }}=\pi-|\Delta \varphi|$ and $\Delta \eta \quad \Delta \varphi$ the relative angles between muon momenta.
RESBOS [15] uses a resummation procedure of leading contributions at the next-to-next-to-leading-logarithms (NNLL) and matches the results to NLO pQCD level calculation, and POWHEG [16] uses a fixed order NLO approximation interfaced to the PYTHIA Parton Shower model. Fig. (4a, b) - where the ratios of predictions over measurements are given - show that the fixed order calculation at NNLO overestimates the data at low $p_{T}$ and $\phi^{*}$, and it underestimates them at high values. On the other hand, RESBOS, which uses a resummation procedure, describes well the data, and the same is true to some extent for POWHEG estimates, which unfortunately lack for now the associated theoretical errors. The FEWZ, RESBOS and 


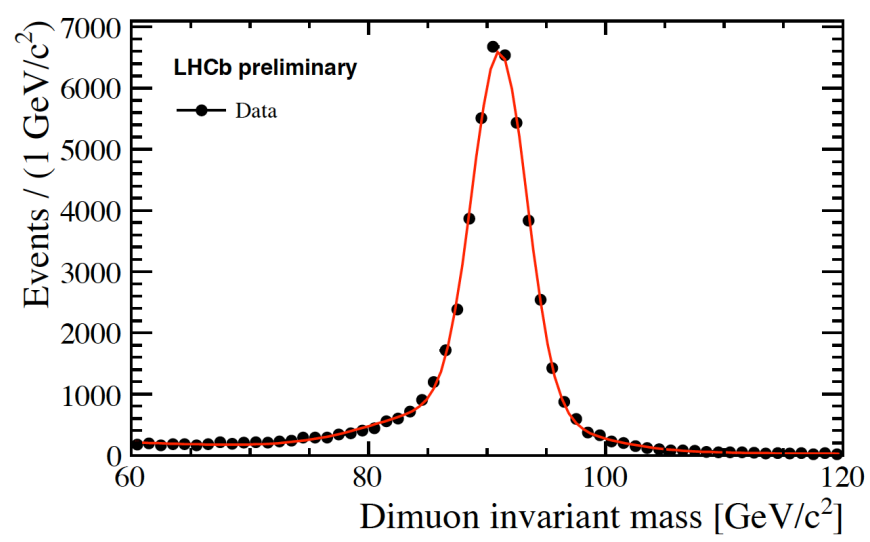

(a)

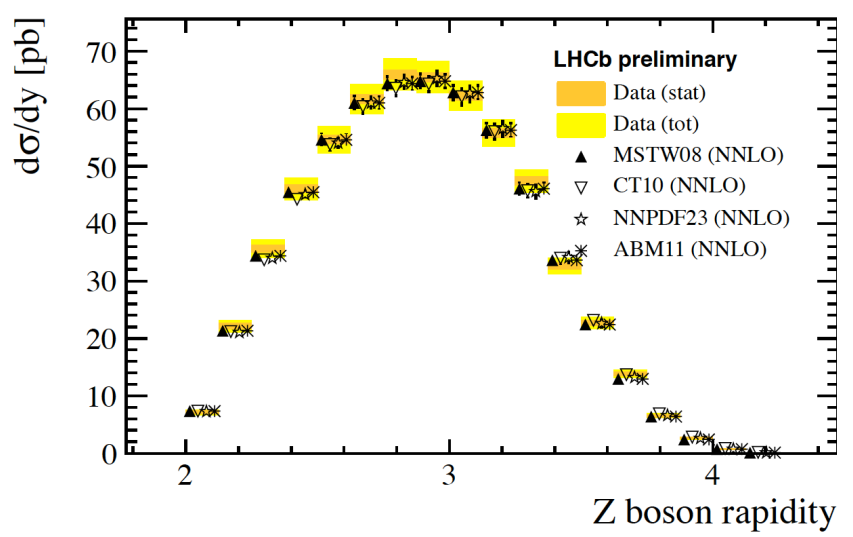

(b)

Fig. (3). Spectrum of dimuon invariant mass for $Z \rightarrow \mu^{+} \mu^{-}$candidates after final selection (a). Measured differential $Z$ cross-section with overlaid theoretical estimates from $\operatorname{FEWZ}(\mathbf{b})$.

POWHEG estimated distribution shapes are normalized to match the $\mathrm{LHCb}$ measured integrated cross-section value.

The low mass Drell-Yan inclusive production crosssection was also measured at $\mathrm{LHCb}$, for $Z / \gamma^{*}$ masses below $60 \mathrm{GeV} / c^{2}$ and down to a minimum of $5 \mathrm{GeV} / c^{2}$. The preliminary results for the integrated cross-section and differential cross-section over dimuon mass and rapidity agree within errors to NLO predictions [17].

\section{INCLUSIVE W PRODUCTION IN THE FORWARD REGION AT $\sqrt{s}=7$ TEV}

The W cross-section measurements [13] are based on 2010 data taken at $7 \mathrm{TeV}$ collision energy with $37 \mathrm{pb}^{-1}$ integrated luminosity. The leptonic decay channel $W^{ \pm} \rightarrow \mu^{ \pm} v(\bar{v})$ is used in the cross-section measurement, by reconstructing the single high $p_{T}$ muon of the final state.

It can be pointed out that with respect to $\mathrm{Z}$ analysis, the fiducial and kinematic cuts on the single muon are same:

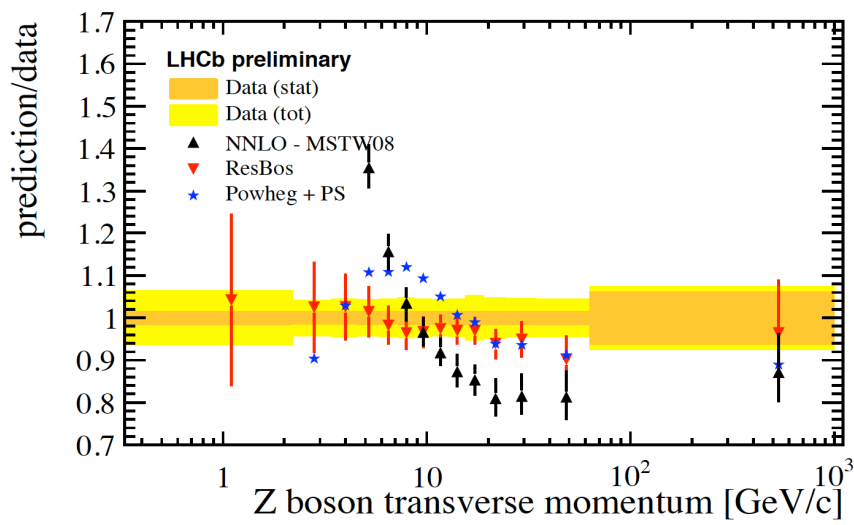

(a)
$p_{T}>20 \mathrm{GeV} / \mathrm{c}$ and $\eta \in[2,4.5]$. A single muon trigger was used in filtering the events, too.

Contrary to the $\mathrm{Z}$ measurement, the $\mathrm{W}$ sample contains a non-negligible fraction of background events. Cuts are imposed on the muon isolation and on the energy deposited in the calorimeters to reduce background. The latter removes most of the background from pions or kaons punching through calorimeter layers. Remaining sources of background are: semileptonic decays of heavy flavored hadrons, decays in flight of pions or kaons, partially reconstructed $Z \rightarrow \mu \mu$, and other $\mathrm{Z} / \mathrm{W}$ decay channels. The background from $Z \rightarrow \mu \mu$ decay is reduced by requiring that there is no other reconstructed muon in the event with $p_{T}>2 \mathrm{GeV} / \mathrm{c}$ and which passes the other muon selection cuts.

The $\mathrm{W}$ yield is extracted by a template fit to the muon $p_{T}$ distribution, simultaneously over five bins in $\eta$. The template for the signal is taken from simulations, and the background shapes from data when possible. Results of the

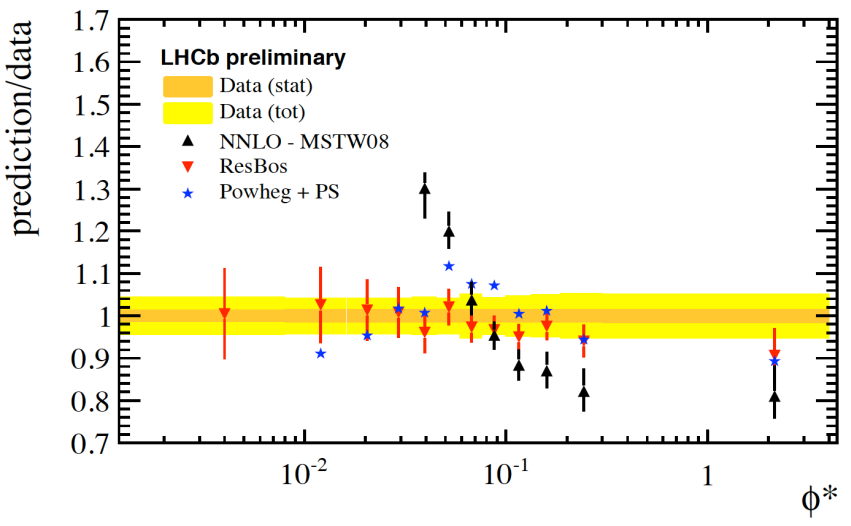

(b)

Fig. (4). Theoretical differential $\mathrm{Z}$ production cross-section divided by the measurements over $p_{T}$ (left) and $\varphi^{*}$ (right). Overlaid are the relative measurement uncertainties: statistical (orange) and systematic (yellow). 


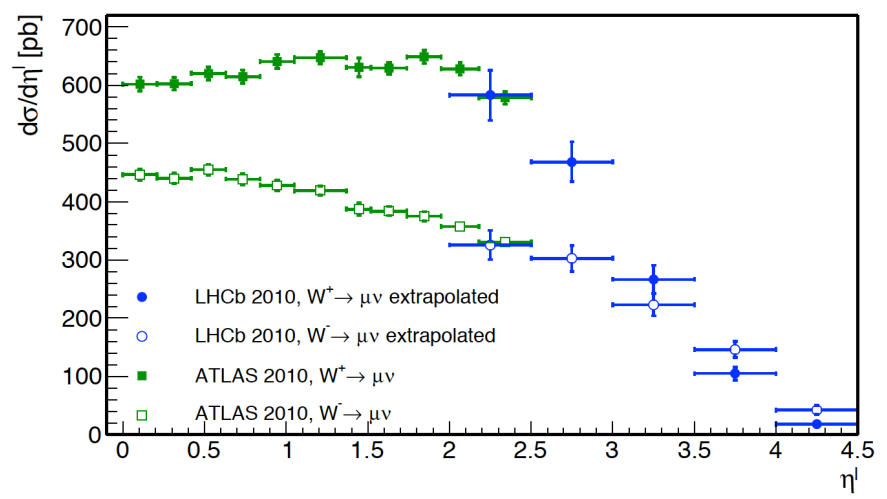

(a)

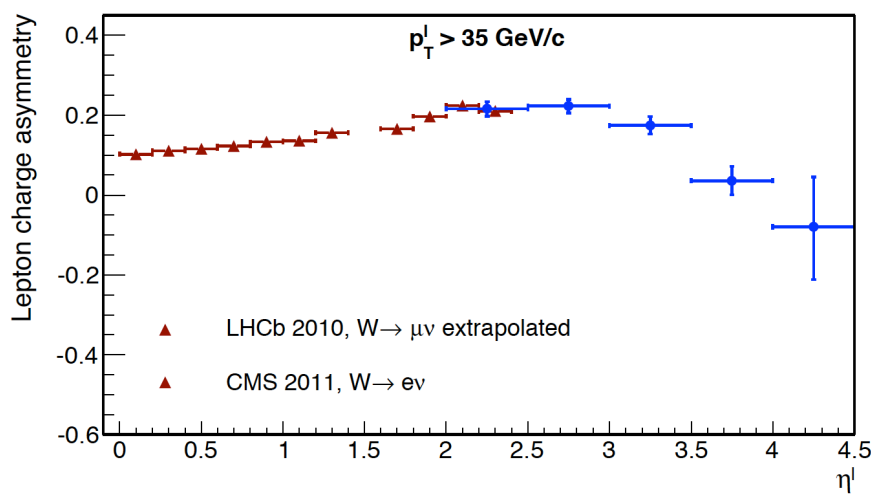

(b)

Fig. (5). Differential production cross-sections for $W^{-}$and $W^{+}$bosons as a function of the lepton pseudorapidity $\eta^{l}$, extrapolated and compared with ATLAS (left). Charge asymmetry as function of lepton pseudorapidity extrapolated and compared with CMS (right).

fit give for $14660 \mathrm{~W}^{+}$and $11618 \mathrm{~W}^{-}$candidates: $44.3 \pm 1.2$ $\%$ purity for $W^{+}$and $34.9 \pm 1.1$ purity for $W^{-}$sample. The cross-sections for the two bosons with the muon in the fiducial and kinematical volume of the measurements are: $\sigma_{W^{+} \rightarrow \mu^{+} v}=831 \pm 9 \pm 27 \pm 29 p b^{-1}$

and

$\sigma_{W^{-} \rightarrow \mu^{-} v}=656 \pm 8 \pm 19 \pm 23 p b^{-1}$

with the first error being statistical, the second systematic, and the third due to the luminosity uncertainty. The systematic uncertainties are on: signal purity, template shape used in fit, tracking and trigger efficiency, selection cuts, muon identification efficiency, and final state radiation corrections. Due to the canceling out of most systematic uncertainties for the ratio of the two cross-sections $R_{W}=\sigma_{W^{+}} / \sigma_{W^{-}}=1.27 \pm 0.02 \pm 0.01$, the total relative error drops to $1.7 \%$ which is comparable to the theoretical uncertainties on the $R_{W}$ fraction. This is an exceptionally precise test on a Standard Model observable. Also, precedent results were compared to NNLO estimates using various PDFs [13] and no significant disagreement was found. The previous $\mathrm{W}$ production results are extrapolated - see reference [18] - to the fiducial volume of the ATLAS measurement. In Fig. (5a) the extrapolated $\mathrm{LHCb}$ results are shown together with the ATLAS results [19] for the $W^{+}$and $W^{-}$differential cross-sections in lepton pseudorapidity. Fig. (5b) shows the CMS lepton charge asymmetry [20]: $\left(\sigma\left(W^{+}\right)-\sigma\left(W^{-}\right)\right) /\left(\sigma\left(W^{+}\right)+\sigma\left(W^{-}\right)\right)$with the extrapolated $\mathrm{LHCb}$ results overlaid. Here, the extrapolation to CMS fiducial implies a cut on the muon transverse momentum at: $p_{T}>35 \mathrm{GeV} / \mathrm{c}$.

\section{SUMMARY AND OUTLOOK}

The energy flow of charged particles for pp collisions at $7 \mathrm{TeV}$ center-of-mass energy was measured and the results were compared to prediction from PYTHIA and cosmic-ray collision generators. Though three of the generators come close to describe the measured energy flow of charged particles over pseudorapidity bins, none of the generators can account for all data features. The $\mathrm{W}$ and $\mathrm{Z}$ boson crosssections were measured by $\mathrm{LHCb}$ at $7 \mathrm{TeV}$ for muon $\eta$ in $[2,4,5], p_{T}>20 \mathrm{GeV} / \mathrm{c}$, and in case of $\mathrm{Z}$ for a mass between $[60,80]$ in $\mathrm{GeV} / c^{2}$. The differential cross-sections were compared with theoretical expected values. While the $\eta$ distribution agree well between theory and measurement, deviations from the observed spectra in $p_{T}$ and $\phi^{*}$ are seen for the fixed order calculations. Predictions including resummation or parton showers agree well with data.

Work is ongoing to finalise the $7 \mathrm{TeV}$ analysis with the full $1 \mathrm{fb}^{-1}$ data set for the $\mathrm{W}$ and the low mass Drell-Yan measurement and to measure $\mathrm{Z}, \mathrm{W}$ and low mass Drell-Yan production at $8 \mathrm{TeV}$ with $2 \mathrm{fb}^{-1}$ which accesses a new kinematic range.

\section{CONFLICT OF INTEREST}

The authors confirm that this article content has no conflicts of interest.

\section{ACKNOWLEDGEMENTS}

We thank the LowX Workshop organizers. And the author acknowledges financial support from: the Romanian Ministry of National Education (MEN) and the Institute of Atomic Physics Bucharest (IFA) under grant number 3/3.01.2012; and UEFISCDI under project grant PN-II-IDPCE-2011-3-0749.

\section{REFERENCES}

[1] Bartalini P, Berger EL, Blok B, et al. Multi-Parton interaction at the LHC. High Energy Phys 2011; ArXiv: 1111.0469 [hep-ph]: 168.

[2] Sjöstrand T, van Zijl M. A multiple-interaction model for the event structure in hadron collisions. Phys Rev D 1987; 36: 2019-41.

[3] LHCb collaboration. The LHCb detector at the LHC. JINST 2008; 3: S08005-205.

[4] Nurse E, Sen S. Methods to select soft diffraction dissociation at the LHC. ArXiv:1107.2688 [hep-ph] 2011; 1-24. 
[5] LHCb collaboration. Measurement of the forward energy flow in pp collisions at $\sqrt{s}=7 \mathrm{TeV}$. Eur Phys J C 2013; 73: 2421-15.

[6] Skands PZ. Tuning Monte Carlo generators: the Perugia tunes. Phys Rev D 2010; 82: 074018-25.

[7] Belyaev I, Brambacht $\mathrm{T}$, Brook $\mathrm{NH}$, et al. Handling of the generation of primary events in Gauss, the $\mathrm{LHCb}$ simulation framework, Nuclear Science Symposium Conference Record (NSS/MIC) IEEE 2010; 1155-61.

[8] Sjöstrand T, Mrenna S, Skands P. A brief introduction to PYTHIA 8.1. Comput Phys Commun 2008; 178: 852-67.

[9] Pierog T, Werner K. EPOS model and ultra high energy cosmic rays. Nucl Phys Proc Suppl 2009; 196: 102-5.

[10] Ostapchenko S. Status of QGSJET. AIP Conf Proc 2007; 928: 11825.

[11] Ahn EJ, Engel R, Gaisser TK, et al. Cosmic ray interaction event generator SIBYLL 2.1. Phys Rev D 2009; 80: 094003-17.

[12] LHCb collaboration, Measurement of the cross-section for $z \rightarrow \mu^{+} \mu^{-}$production with $1 \mathrm{fb}^{-1}$ of pp collisions at $\sqrt{s}-7 \mathrm{TeV}$. LHCb-CONF-2013-007.

[13] LHCb collaboration, Inclusive $\mathrm{W}$ and $\mathrm{Z}$ production in the forward region at $\sqrt{s}=7$ TeV. J High Energy Phys 2012; 06: 058-29.

[14] Gavin R, Lia Y, Petriello F, et al. FEWZ 2.0: A code for hadronic $\mathrm{Z}$ production at next-to-next-to-leading order. Comput Phys Commun 2011; 182: 2388-403.

[15] (a) Ladinsky GA, Yuan CP. The Nonperturbative Regime in QCD Resummation for Gauge Boson Production at Hadron Colliders.
Phys Rev D 1994; 50: R4239-R4243. (b) Balazs C, Yuan CP. Soft gluon effects on lepton pairs at hadron colliders. Phys Rev D 1997; 56: 5558-5583. (c) Landry F, Brock R, Nadolsky PM, et al. Tevatron Run-1 Z Boson Data and Collins-Soper-Sterman Resummation Formalism. Phys Rev D 2003; 67: 073016-8.

[16] (a) Nason P. A new method for combining NLO QCD with shower Monte Carlo algorithms. J High Energy Phys 2004; 0411: 040-27.

(b) Frixione S. Nason P, Oleari C. Matching NLO QCD computations with Parton shower simulations: the POWHEG method. J High Energy Phys 2007; 11: 070-91. (c) Alioli S, Nason $\mathrm{P}$, Oleari $\mathrm{C}$, et al. A general framework for implementing NLO calculations in shower Monte Carlo programs: the POWHEG BOX. J High Energy Phys 2010; 06: 043-56.

[17] LHCb collaboration. Inclusive low mass Drell-Yan production in the forward region at $\sqrt{s}=7 \mathrm{TeV}$. LHCb-CONF-2012-013.

[18] LHCb collaboration. Graphical comparison of the $\mathrm{LHCb}$ measurements of $\mathrm{W}$ and $\mathrm{Z}$ boson production with ATLAS and CMS. LHCb-CONF-2013-005.

[19] ATLAS collaboration. Measurement of the inclusive $W^{ \pm} \mathrm{Z} / \gamma^{*}$ and cross-sections in the electron and muon decay channels in pp collisions at $\sqrt{s}=7 \mathrm{TeV}$ with the ATLAS detector. Phys Rev D 2012; D85: 072004-39.

[20] CMS collaboration. Measurement of the electron charge asymmetry in inclusive $\mathrm{W}$ production in pp collisions at $\sqrt{s}=7$ TeV. Phys Rev Lett 2012; 109: 111806-15. 